\title{
Examining gap between planned service levels and realistic demands of hospitals based on accessibility driven service area demarcation an empirical study of Dalian, P.R. China and Berlin, Germany
}

\author{
Xiang AO, Shanghai Tongji Urban Planning and Design Institute, China \\ Qianwen LI, Shanghai Tongji Urban Planning and Design Institute, China
}

\begin{abstract}
The appropriate allocation and service level configuration of hospitals is of great importance in improving the quality of individuals' life, as well as maintaining social security and social stability. Yet in traditional urban planning projects in China, hospitals are usually roughly configured and laid where the population of certain districts exceeds 1,000, i.e, according to an index of every one thousand persons, or where the residents of nearby communities could reach in 15 minutes by walk, i.e, according to standards of 15-minute life circle. Crude hospital configuration methods as such render potential gap between planned supplies and realistic demands of medical resources. Previous researches addressing such gap often rely on ex ante accessibility analysis such as network analysis via Geographic Information System, often failing to take into considerations realistic factors like traffic congestions, enclosures, and thus not precisely reflecting the actual travel time. To overcome the above defects, this study applies an ex post accessibility method, relying on online map providers to directly acquire real travel time. Firstly, Berlin is chosen as the comparison city for Dalian to draw guidance on hospital distribution due to its high development level and its similarities to Dalian in population and area. Secondly, grid points covering whole urban areas are generated for each city, and the travel time between each grid point and all hospitals are acquired from online map providers. Grid points are then assigned to hospitals with the shortest travel time, and realistic hospital service areas are thus demarcated. Thirdly, population distribution data are overlaid with realistic hospital service areas. Thus, it can be inferred to what amount of residents each hospital is burdened with, and whether this amount is appropriate given with the planned service level of each hospital. Finally, the results of Dalian are compared with Berlin, showing there is more apparent disparity in hospital spatial accessibility, and notable gap between planned service level and realistic demands of hospitals in Dalian. Discussions are later made to provide concrete suggestions for improved hospital allocation and configuration for Dalian. This research contributes to the expanding literature on hospital allocation and configuration in raising a novel method applicable for inference of the actual hospital service areas and service burden.
\end{abstract}

\section{Keywords}

public facility, spatial accessibility, geographic information system, urban data analytics

\section{Introduction}

The scientific and rational allocation of hospitals is appreciable for realizing the maximization of social benefits and welfare, promoting people's livelihood and health, improving the living environment, as well as maintaining social equity (Song et al., 2010). Since the 1990s, health city programs have gradually draw 
the attention of Chinese urban planners and scholars. The public medical service level and its spatial accessibility were broadly applied as the key research indicators in related study (Lan et al., 2016). Furthermore, in the context of post-EPIDEMIC era, how to timely respond to public health emergencies has also become a hot topic. China has engaged in exploring a sustainable development path for its urban medical service system. Therefore, study on the appropriate allocation and service level configuration of hospitals is definitely conducive to comprehensively improving the level of medical service allocation in China. It is not only helpful for theoretically understanding the status quo of urban medical resources and identifying medical shortage areas, but can also give guidance for optimizing the spatial layout of medical facilities in practice (Tao and Shen).

The traditional methods to configure medical facilities in China include: the number of technical personnel and beds are calculated in proportion with the index of 1,000 people (Zhang et al., 2006). As the living circle theory has gradually been gaining focus in Chinese urban planning programs (Meng, 2017, Rong, 2018), there is also alternative way to configure medical service centers so that residents can reach the nearest one within 15 minutes. Yet, traditional methods often suffer from a simplistic estimation of medical service demands, and thus rendering inconsistency with the reality. For example, the method to configure medical facilities based on index of 1,000 often can be easily undermined due to a negligence of population mobility factors, if we merely consider from the perspective of population (Chai and Li, 2019). As of the method to configure medical facilities based on 15-minute living circle, medical facilities are often simplistically laid where nearby communities are not farther than certain euclidean distance, and thus overlooking the realistic accessibility (Zheng et al., 2019).

Thus, this study aims to fill some of the gaps by introducing a novel mothod of evaluating differences between planned service levels and realistic demands of general hospitals based on accessibility driven service area demarcation. The method starts from the following hypotheses: firstly, we take the available beds as reflection of planned service level of certain hospitals, and the population that resides within the service area can be seen as reflection of realistic demands of certain hospitals. Secondly, we assume that normally patients would choose a general hospital with the shortest travel time. Thus, the service area of certain general hospital should be so demarcated, that from anywhere in the service area the realistic travel time to that very hospital should be the shortest as compared to all other general hospitals.

\section{Data and methods}

\subsection{Study areas}

Dalian is a livable tourist coastal city located in Liaoning Province. Since the launch of China's Reform and Opening Up policy in 1980s, Dalian has rapidly grown into one of the most prosperous coastal cities in China. As the economic center of Liaoning Province, its annual GDP reached over 703 billion RMB (approximately 92 billion Euro) in 2020, ranking the first among cities in Northeastern Region of China. It has a population of over 7.4 million, and an area of over 12,500 square kilometers. The concept of "Dalian City" actually involves an inclusion of both urban area and rural area. The urban area of Dalian consists of four districts, namely Zhongshan District, Xigang District, Shahekou District and Ganjingzi District, totaling a population of over 2.8 million and an area of over 550 square kilometers. To facilitate a direct comparison of Dalian and Berlin, we here focus only on the urban area of Dalian ${ }^{1}$ and thus exclude an analysis of the rest rural area.

Berlin is the capital city and the most populous city of Germany, with a population of over 3.4 million and an area of over 890 square kilometers. With a high economic development level, Berlin led Dalian with a

\footnotetext{
1 "Urban area of Dalian" is referred to as "Dalian" hereinafter for convenience.
}

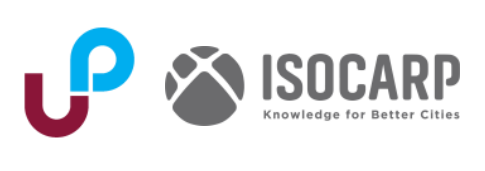


total GDP of over 154 billion Euro in 2020. Moreover, Berlin has a widespread reputation for its top-class medical resources and services with a variety of hospitals and a broad range of health care services.

Our study applies a comparison study between the urban area in Dalian, China, and Berlin, Germany to draw guidance for a more scientific planning of hospital facilities in Dalian. The reasons as to select the above pair city as research object are mainly twofold: 1) Dalian and Berlin have similarities in terms of population and area. Hence, the overall demand and performance of hospitals in Dalian and Berlin should have a good basis for comparison. 2) Berlin exhibits a high development level in medical resources and services. Therefore, Berlin should function well as a model city for Dalian to draw guidance on improving the planning of hospital facilities.

\subsection{Data sources}

Firstly, the data on general hospitals in Berlin are extracted from the registered association Berlin the City of Health ${ }^{2}$ (Der Gesundheitsstadt Berlin e. V.), which is a non-profit association with the aim to "promote public health care in the Berlin-Brandenburg region through events and information". Berlin the City of Health offers the list of hospitals in Berlin along with their addresses and numbers of available beds. In the end, information of a total of 56 hospitals was obtained, and the coordinate of each hospital was acquired via Google Maps API. As of Dalian, a list of tertiary hospitals was obtained from Health Commission of Liaoning Province ${ }^{3}$, and the addresses along with number of available beds were acquired through individual search at the official websites. The hospitals in China are classified into primary hospitals, secondary hospitals and tertiary hospitals. According to relevant regulations, only tertiary hospitals bear the responsibility of "providing comprehensive medical and health services", while for hospitals of other grades the functions mainly include "providing prevention measures", "monitoring groups at high risks", etc. Hence, we focus only on the tertiary hospitals in Dalian for a unified calibration of hospitals with Berlin. Finally, information of a total of 22 hospitals was obtained, and the coordinates were acquired via AMAP Maps API. A visualization of the distribution of hospitals and number of available beds in both Dalian and Berlin can be found in Figure 1.

Secondly, the travel time between any origins and hospitals is needed for service area demarcation. Unlike previous studies where the travel time is estimated by modelling the travel distance and speed, we here directly make requests on the realistic travel time to online map providers, i.e., AMAP Maps and Google Maps.

Thirdly, we utilized the spatial population distribution dataset from Bondarenko et al. (2020) to estimate the overall population within the service area of each hospital in Dalian and Berlin. A visualization of the spatial population distribution in Dalian and Berlin can be found in Figure 2.

\footnotetext{
${ }^{2}$ https://www.gesundheitsstadt-berlin.de/berlin/kliniken/A/, last visited on 10 Sep., 2021

3 http://wsjk.In.gov.cn/, last visited on 6 Sep., 2021
} 

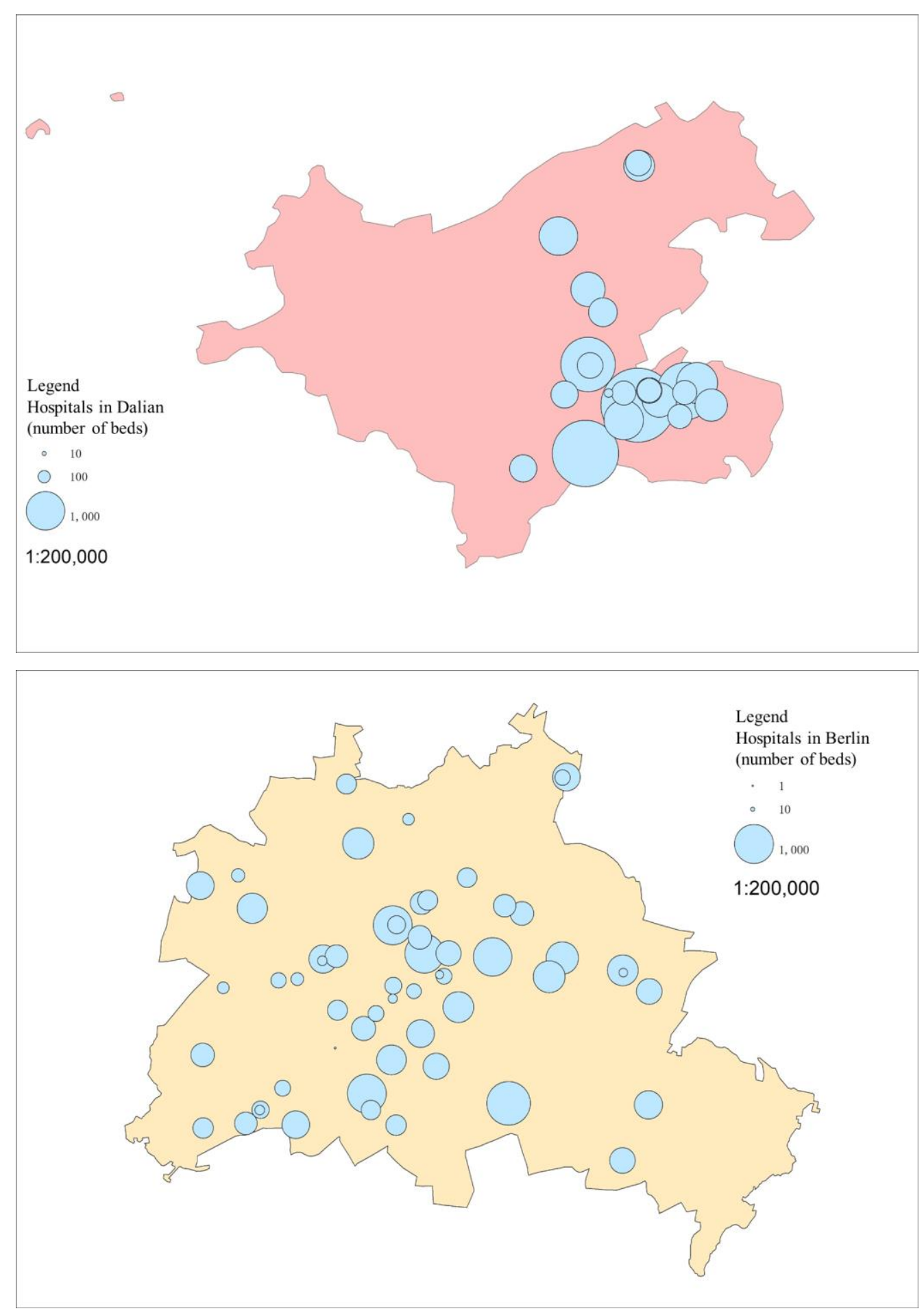

Figure 1 Spatial distribution of hospitals in Dalian and Berlin. Source: Health Commission of Liaoning Province and Berlin the City of Health. 

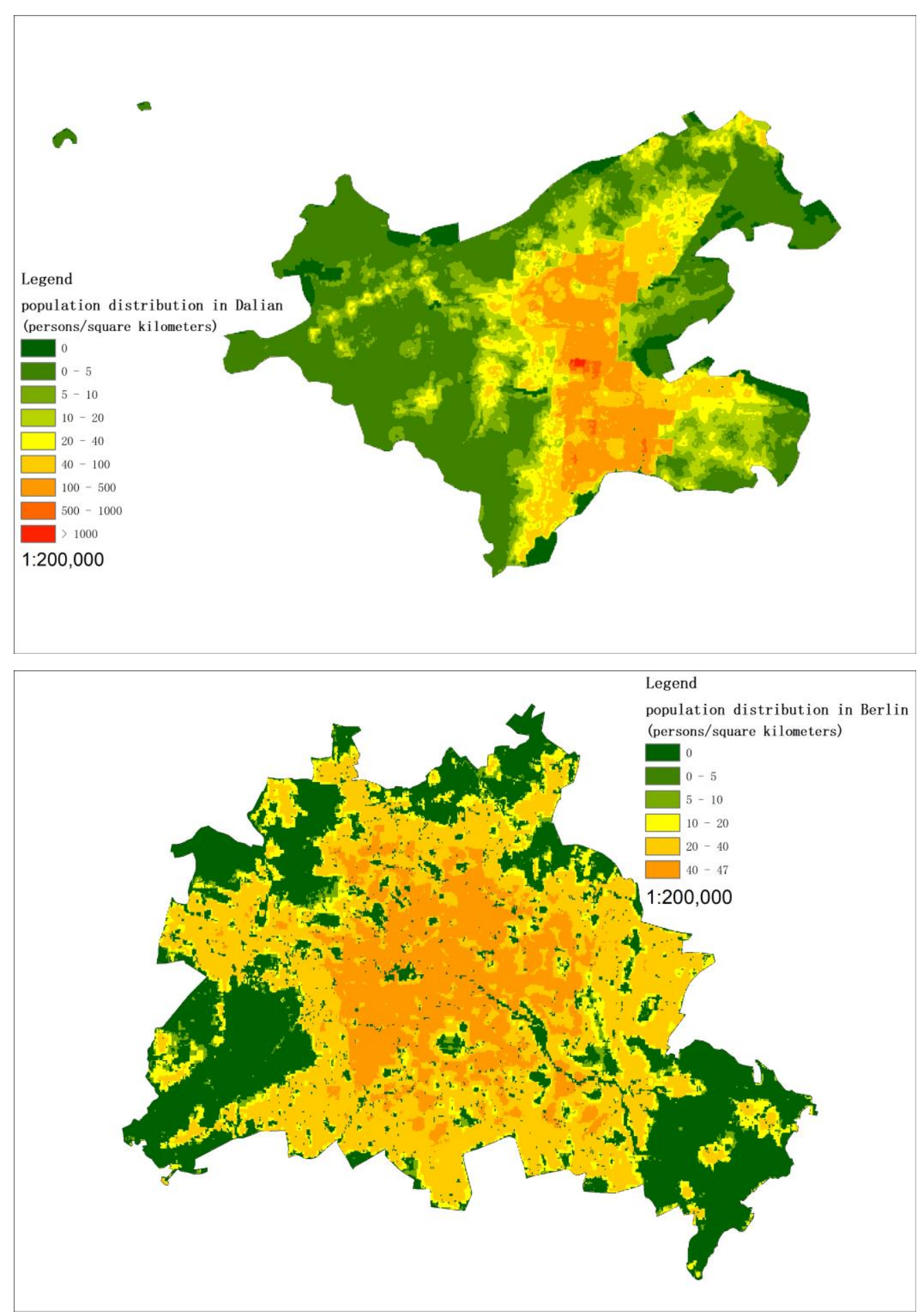

Figure 2 Spatial distribution of population in Dalian and Berlin. Source: Bondarenko et al. (2020)

\subsection{Hospital service area demarcation}

In this study, we propose a novel method to demarcate the hospital service area based on the accessibility defined by the realistic travel time. The basic idea behind this method is as follows: the service area of a certain hospital should be so demarcated, that from anywhere within the service area the realistic travel time should be the shortest to that very hospital compared to other hospitals. 
On that basis, we design the following technical processes: a) grids and centroid preparation: both fishnets covering the whole areas of Dalian and Berlin are generated via Geopandas module in Python. The resolution of the grids in fishnets is specified to $500 \mathrm{~m}$ for convenience of calculation. Then the unit centroid of each grid is then generated, and the coordinate of each unit centroid is extracted via Arcgis. In the end, fishnets of 2417 grids/unit centroids and of 3562 grids/unit centroids are generated for Dalian and Berlin (see Figure 3). b) coordinates of hospitals preparation: requests of hospitals in both Dalian and Berlin are sent via AMAP Maps and Google Maps API, and responses on coordinates of hospitals are obtained in return. c) OD (Origin-Destination) travel time preparation: the realistic travel time is requested between each unit centroid (i.e., origins) and all hospitals (i.e., destinations) again via AMAP Maps and Google Maps API. Considering the hospitals' nature of being public facility, its accessibility then should be measured from the perspective of public transportation. In practice we utilized the navigation mode of AMAP Maps and Google Maps API, and set the travel mode to public transportation, in order to extract the real travel time by public transportation between origins and destinations. d) OD matrix preparation: each unit centroid is assigned to that hospital which records the shortest travel time to form a OD pair. And all OD pairs together consist the OD matrix. e) hospital service area demarcation: based on the OD matrix, all grids are assigned to hospitals with the shortest travel time according to the unit centroids with the same object ID. Thus, all grids that are assigned to the same hospital make up the service area of that hospital.

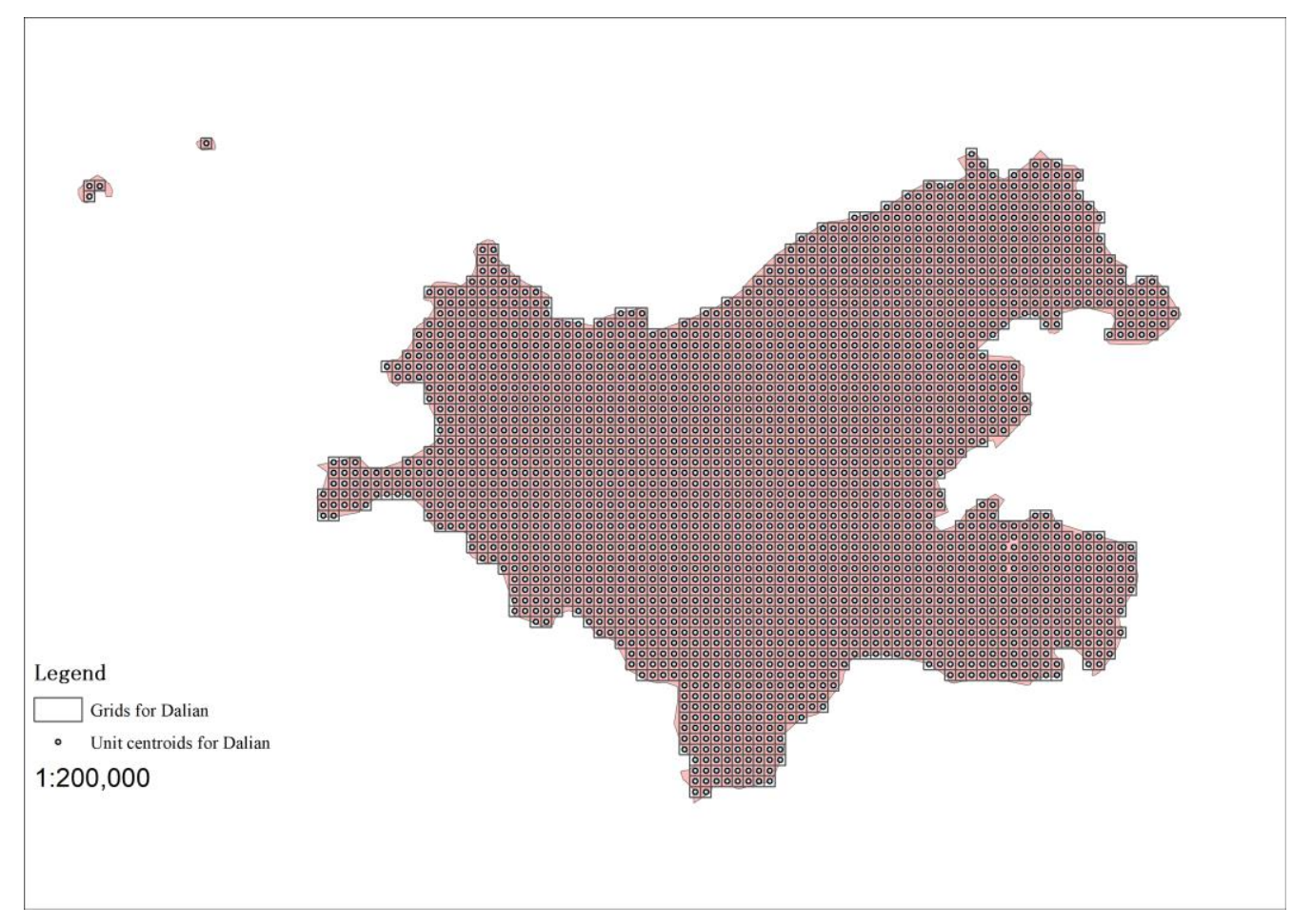




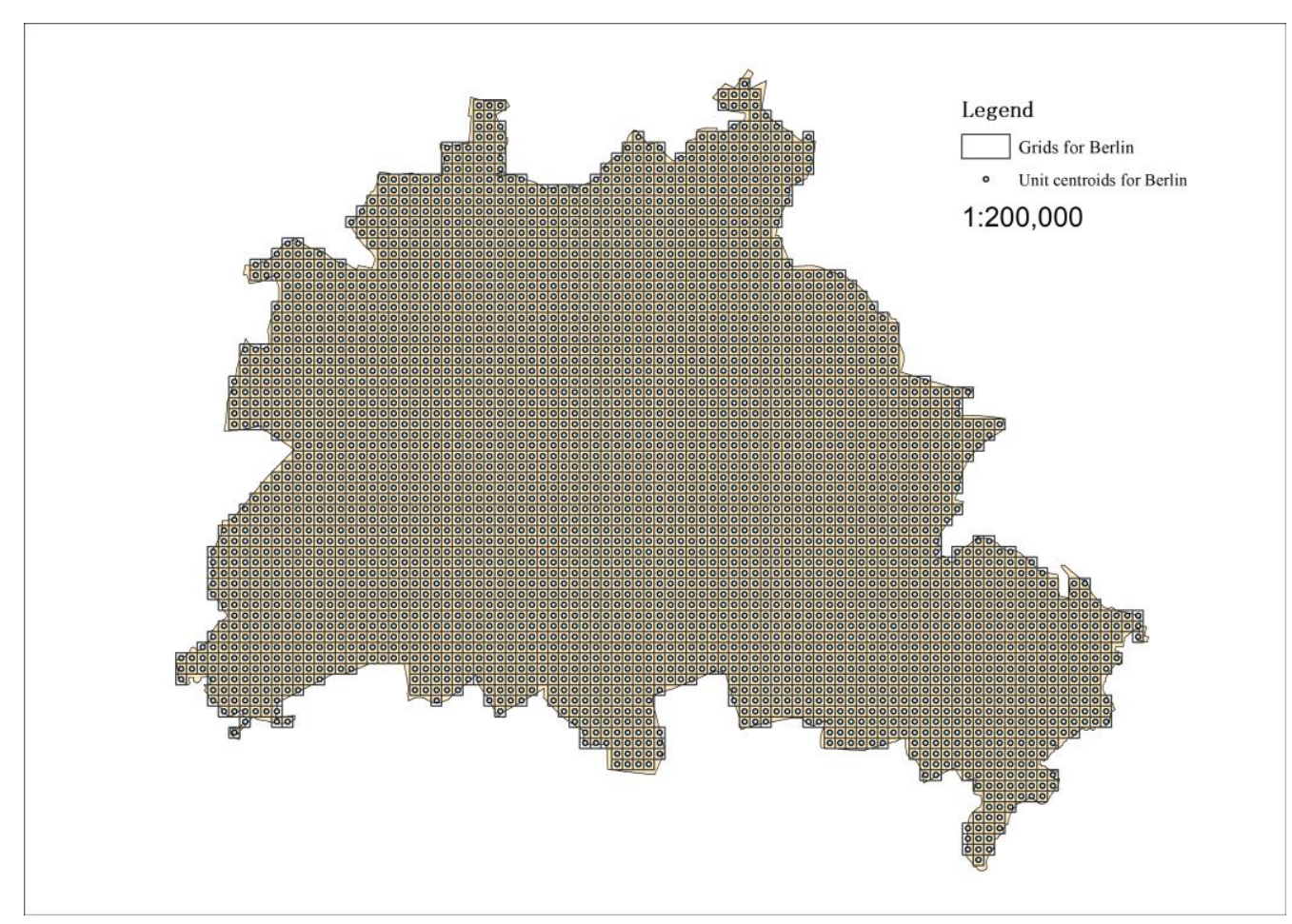

Figure 3 Grids and unit centroids generated for Dalian and Berlin.

\section{Results}

\subsection{General characteristics}

The summary of general characteristics starts with a comparison of number of medical facilities in Dalian and Berlin. It can be shown in Table 1 that the general hospitals in Berlin are twice as many as in Dalian. Yet in terms of total number of beds, Dalian overtakes Berlin with over 21.6 thousand beds to 21.2 thousand beds. Thus, the number of beds per one thousand persons in Dalian reaches 77.3 , a little higher than 62.2 in Berlin. It is then argued that although Dalian offers as many beds as in Berlin, or even more beds per capita, Dalian has far fewer hospitals than Berlin, indicating the most beds are centered in a small number of hospitals. Such argument could be further validated from the observation of beds distribution among hospitals in Dalian and Berlin (see Figure 4). It is concluded that over half of hospitals (55.36\%) in Berlin are equipped with 100 to 500 beds, and less than $10 \%$ of hospitals are equipped with more than 1000 beds. However, in Dalian there are only less than one third of hospitals (31.82\%) with number of beds ranging from 100 to 500 , and over $30 \%$ of hospitals have more than 1000 beds.

Secondly, the spatial distribution of hospitals in Dalian and Berlin is compared. Standard Deviational Ellipse (SDE), an Ellipse with the mean center being the mean center of all input points, the long axis and the short axis being the largest and smallest standard deviation (Scott and Janikas, 2010), is applied here to explore to what extend are medical facilities centered in Dalian and Berlin. We utilized the Directional Distribution tool in Arcgis and set the weight to the number of beds in each hospital to generate SDEs with one standard deviation. As the results in Figure 5 indicate, the SDE in Dalian occupies an area of 87.38 square kilometers, whereas in Berlin that number goes up to 291.51 square kilometers. The difference in the areas of SDEs shows that the medical facilities are distributed much more dispersedly in Berlin than in Dalian. This observation suggests that medical services in Dalian may suffer from a more serious spatial disparity than in Berlin. 
Table 1. Comparison of number of medical facilities in Dalian and Berlin. Source: Health Commission of Liaoning Province and Berlin the City of Health.

\begin{tabular}{lll}
\hline & Dalian & Berlin \\
\hline Total number of general hospitals & 22 & 56 \\
Total number of beds & 21,633 & 21,162 \\
Number of beds per 1,000 persons & 77.3 & 62.2 \\
\hline
\end{tabular}

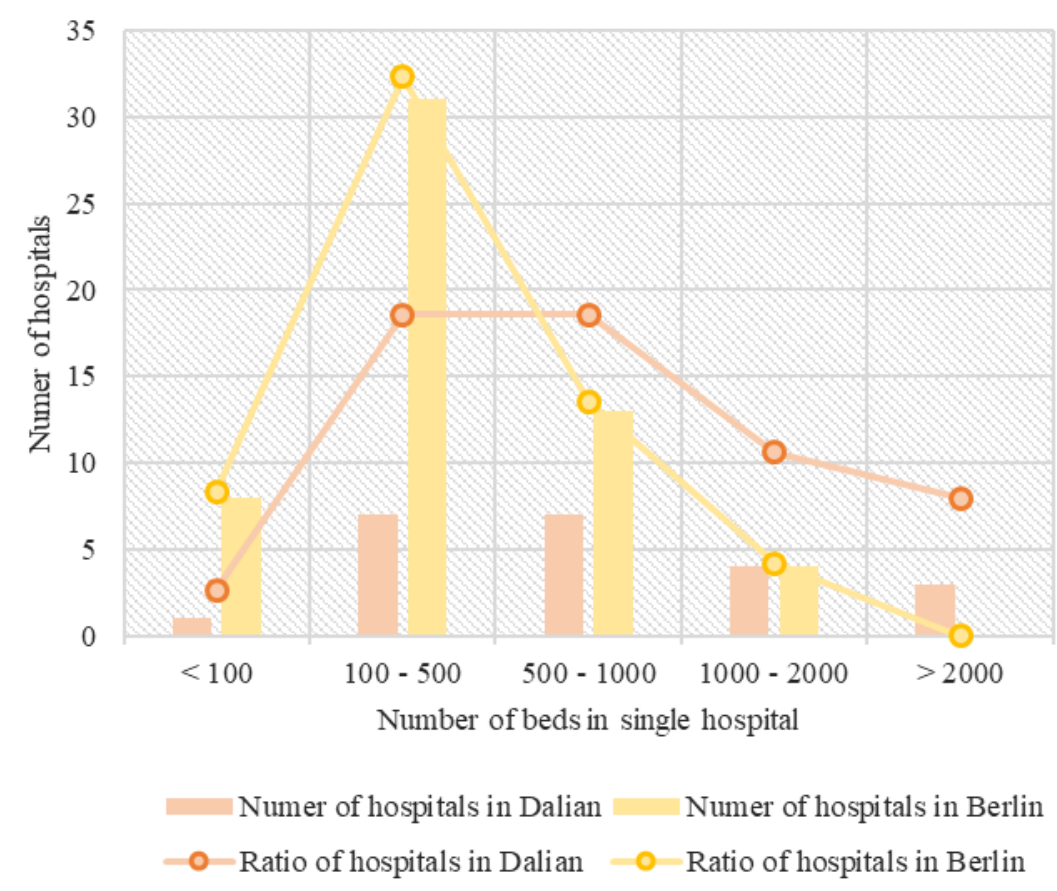

$60 \%$

$50 \%$

$40 \% \frac{\text { a }}{30 \%}$

$10 \%$

$0 \%$

Figure 4 Comparison of bed distribution among hospitals in Dalian and Berlin. Source: Health Commission of Liaoning Province and Berlin the City of Health.

Legend

- Hospitals in Dalian

Standard deviational ellipse

$1: 200,000$
○

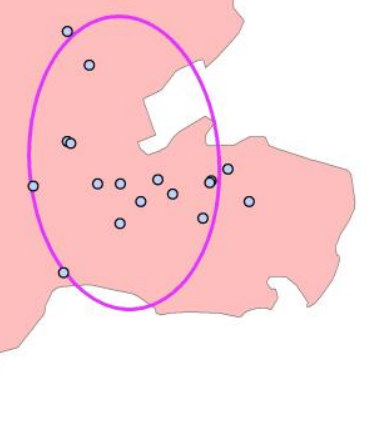




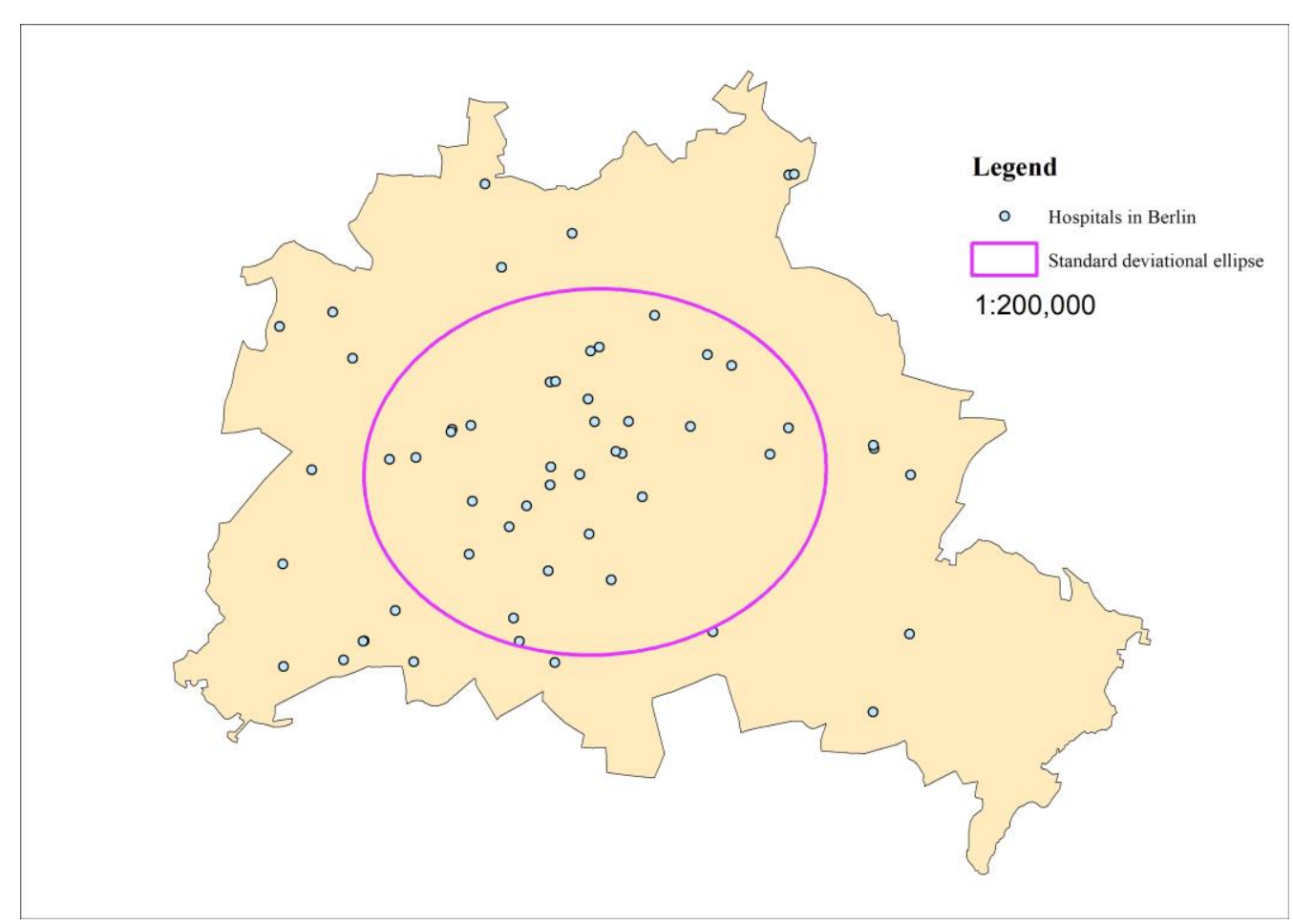

Figure 5 Standard deviatioinal ellipses of hospitals in Dalian and Berlin.

\subsection{Hospital accessibility}

The hospital service areas in Dalian and Berlin are demarcated as in Figure 6. A summary of areas of hospital service areas is given in Figure 7. It can be shown from the summary that the hospital service areas in Dalian on average occupies 27.5 square kilometers, approximately 1.7 times of that in Berlin (15.9 square kilometers), suggesting that larger hospital service areas and thus a potentially poorer hospital accessibility in Dalian. Moreover, the hospital service areas in Dalian have a rather high upper quartile and a rather low lower quartile, indicating a rather fierce spatial disparity on hospital service areas in Dalian.

The distribution of travel time to nearest hospital in Dalian and Berlin is visualized in Figure 8. A summary of travel time to nearest hospital is then given in Figure 9. It can be seen from the summary that the travel time to nearest hospital is in general far larger in Dalian than in Berlin, with the average number in Dalian (69.1 minutes) being nearly three times of that in Berlin (24.7 minutes). Moreover, the lower quartile of travel time to nearest hospital in Dalian (37.7 minutes) is even higher than upper quartile of that in Berlin. The above observations again point to a potentially poorer hospital accessibility in Dalian.

Worth noticing is, despite all the above analyzes on the serious spatial disparity of medical facilities distribution in Dalian, it can still be argued that this so-called disparity is due to the fact that Dalian is a mountainous city where large areas are covered with hills and thus sparsely populated. Thus, we will discuss in the next section the matching degree between planned service levels and realistic demands via overlay of population distribution data. 


\section{Legend}

- Hospitals in Dalian

Service areas

$1: 200,000$
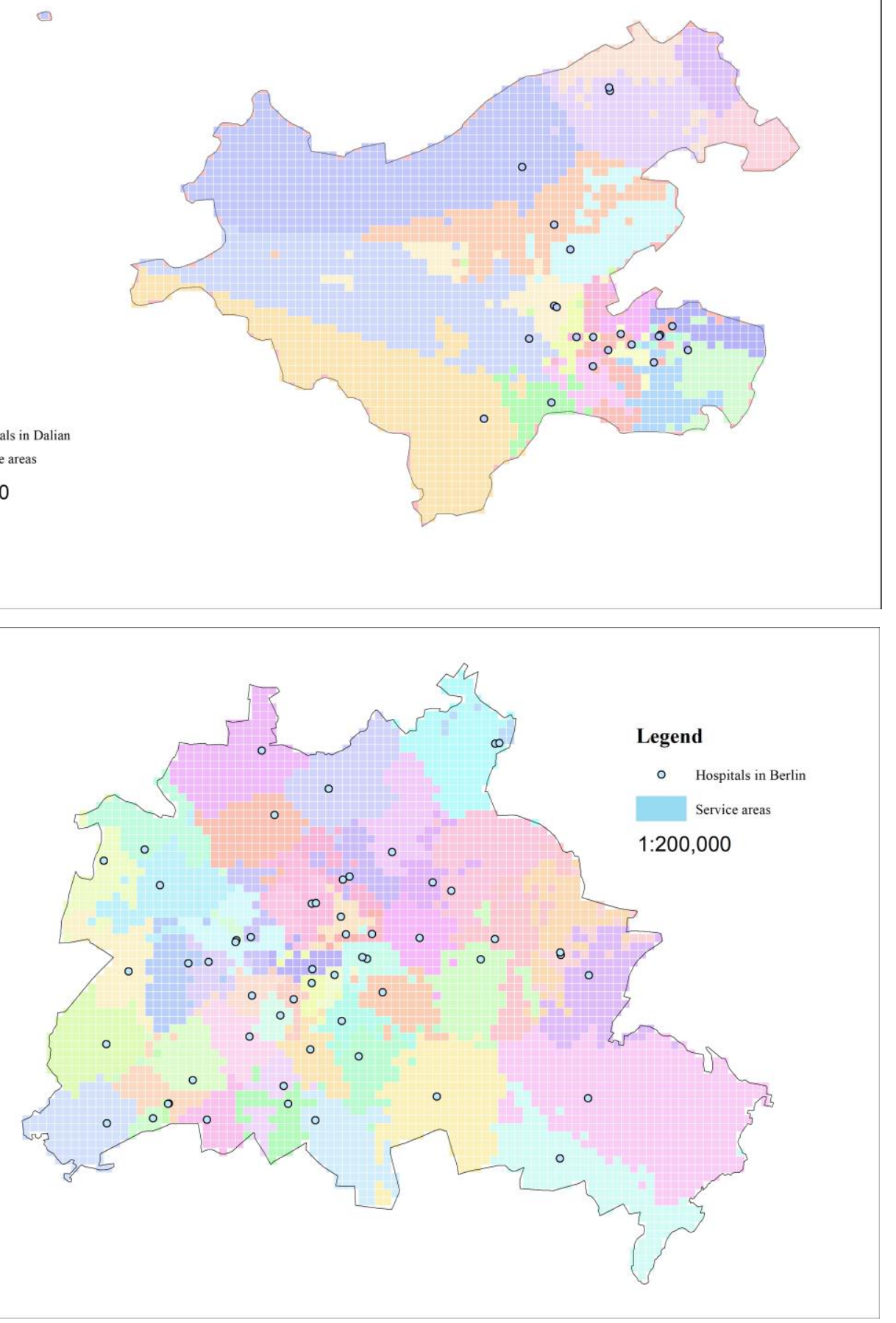

Figure 6 Demarcation of hospital service areas in Dalian and Berlin. 


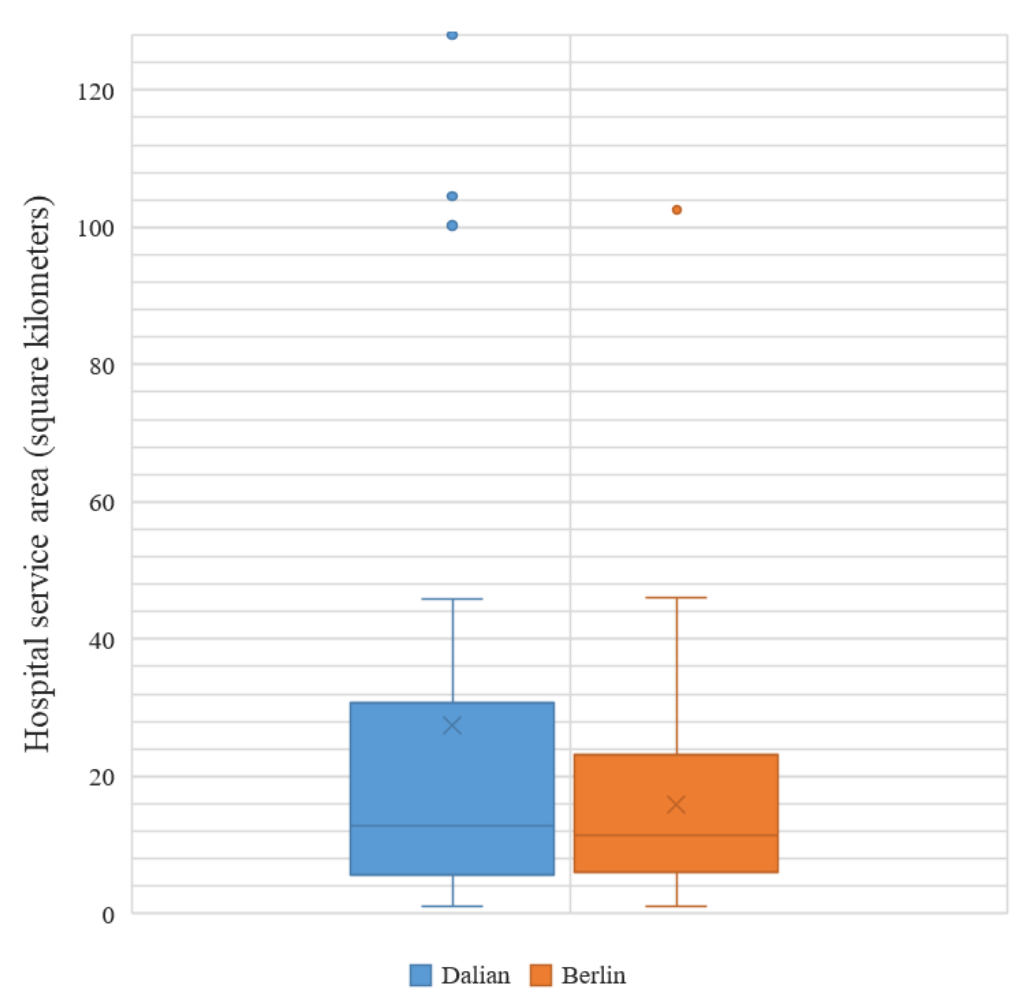

Figure 7 Box plot of hospital service areas in Dalian and Berlin.

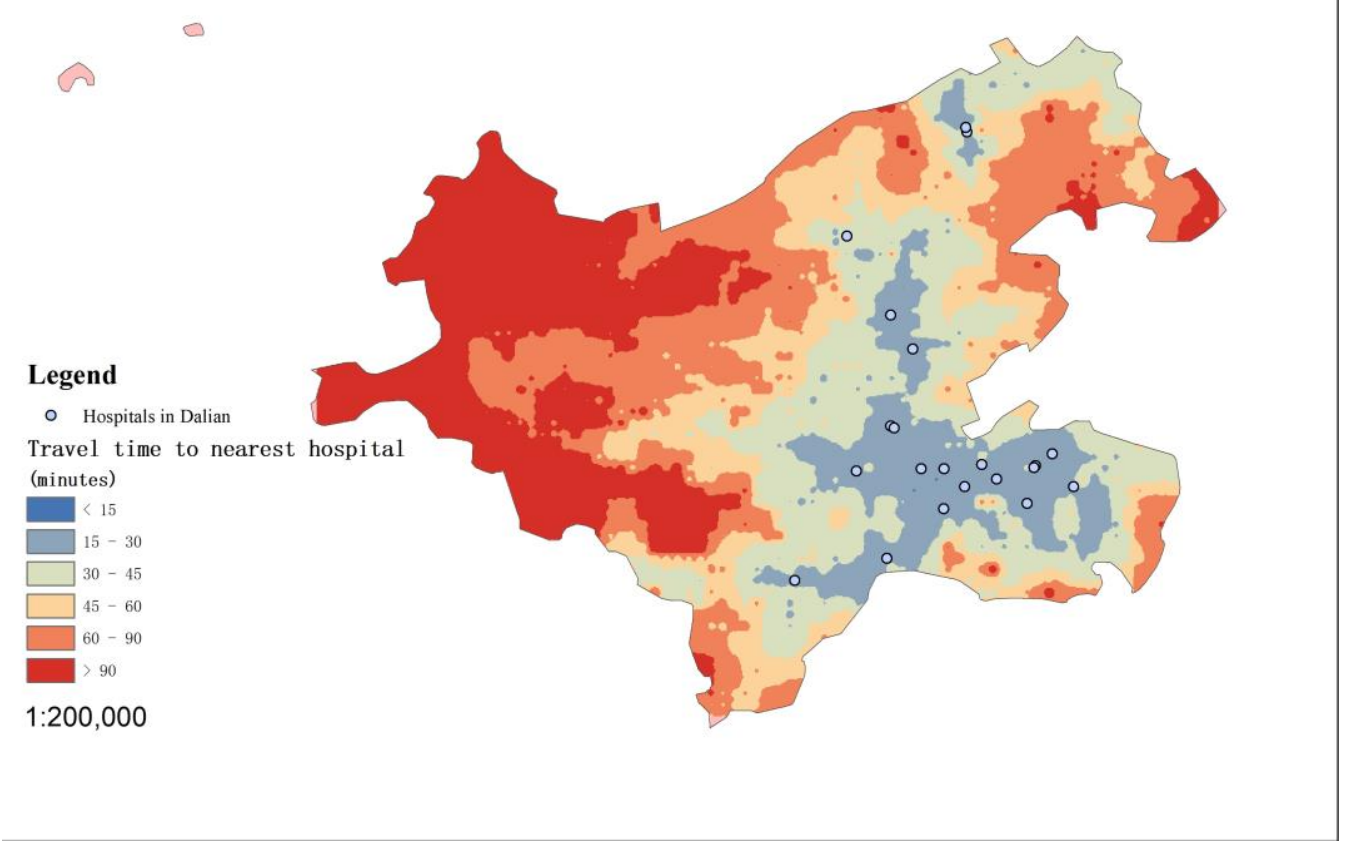




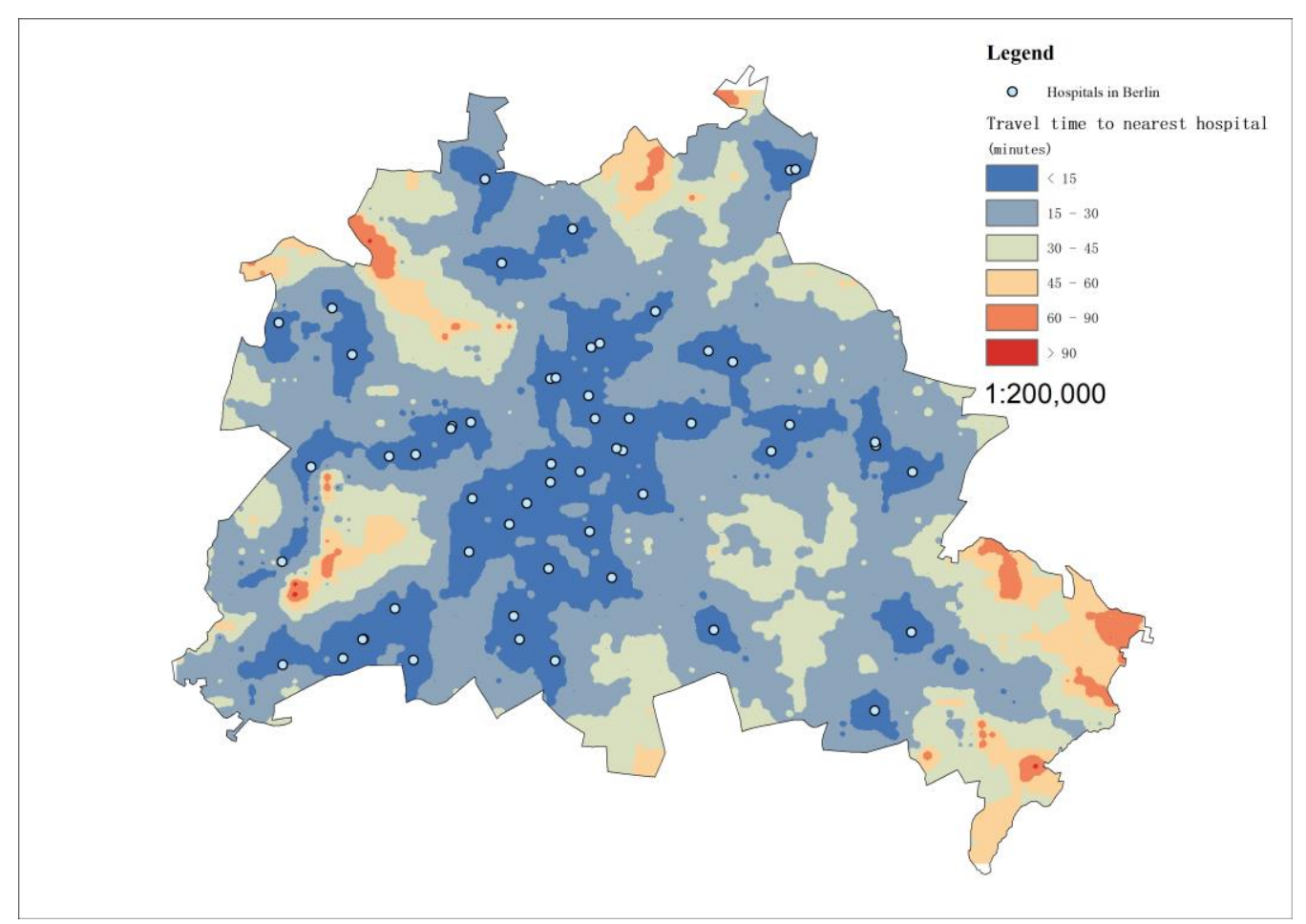

Figure 8 Travel time to nearest hospital in Dalian and Berlin.

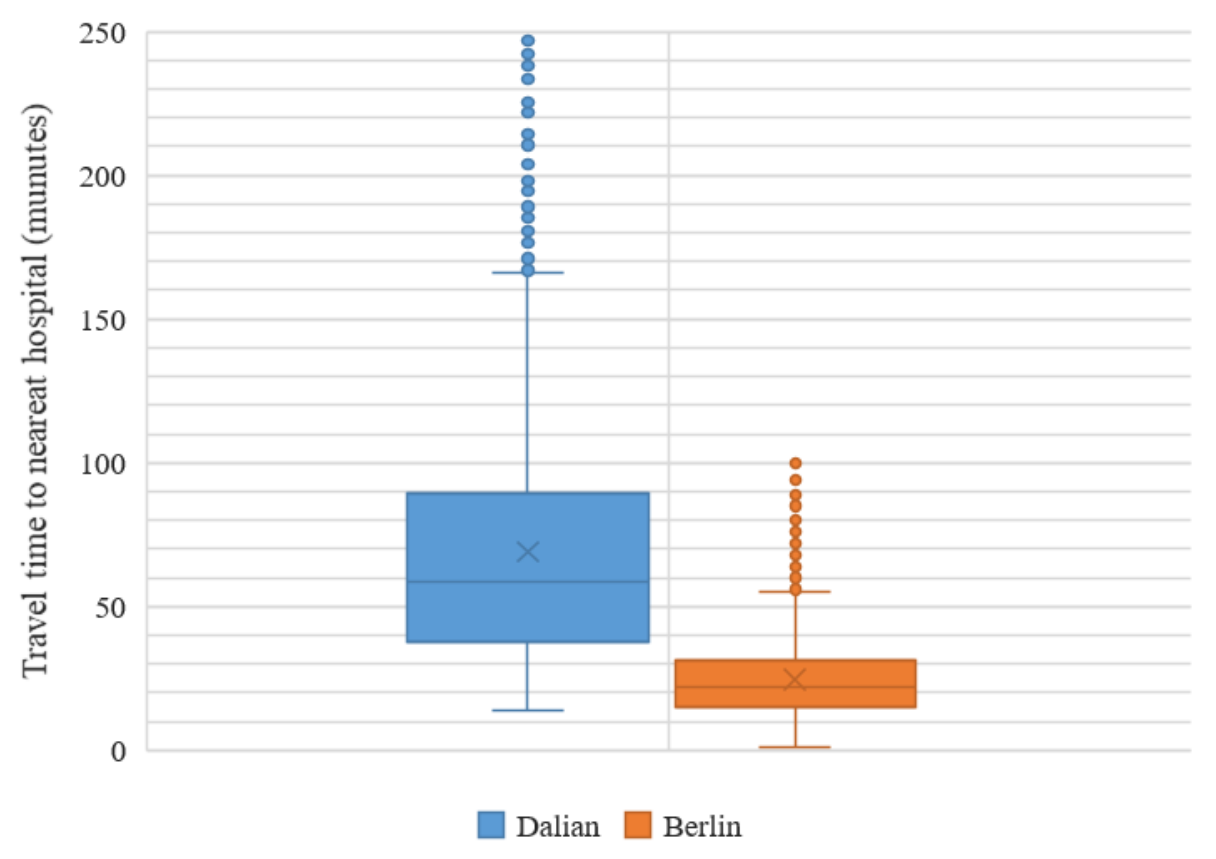

Figure 9 Box plot of travel time to nearest hospital in Dalian and Berlin.

\subsection{Gaps between planned hospital service levels and realistic demands}

We apply here the ratio between the population residing in the hospital service areas and the available beds as the "gap ratio" to explore the gaps between planned hospital service levels and realistic demands. A visualization of "gap ratio" in Dalian and Berlin can be found in Figure 10. It should be noticed that the "gap ratio" should neither be too high, indicating the service level falls far behind the realistic demands, nor too low, indicating the service level is not fully utilized. To facilitate a direct comparison, we calculate the population weighted average "gap ratio", and a Gini coefficient of "gap ratio" in both Dalian and 
Berlin (see Table 2). It can be drawn from the comparison that on average fewer people (every 253 persons) in Berlin would have to "share" a hospital bed than in Dalian. Besides, the Gini coefficient in Dalian (53.1\%) is slightly higher than in Berlin (52.7\%), indicating a more notable gap in planned service levels and realistic demands in Dalian. It is argued that though the total available beds and population are similar in Dalian and Berlin, Berlin managed to keep a relatively low spatial disparity and narrowed gaps between planned service levels and realistic demands.
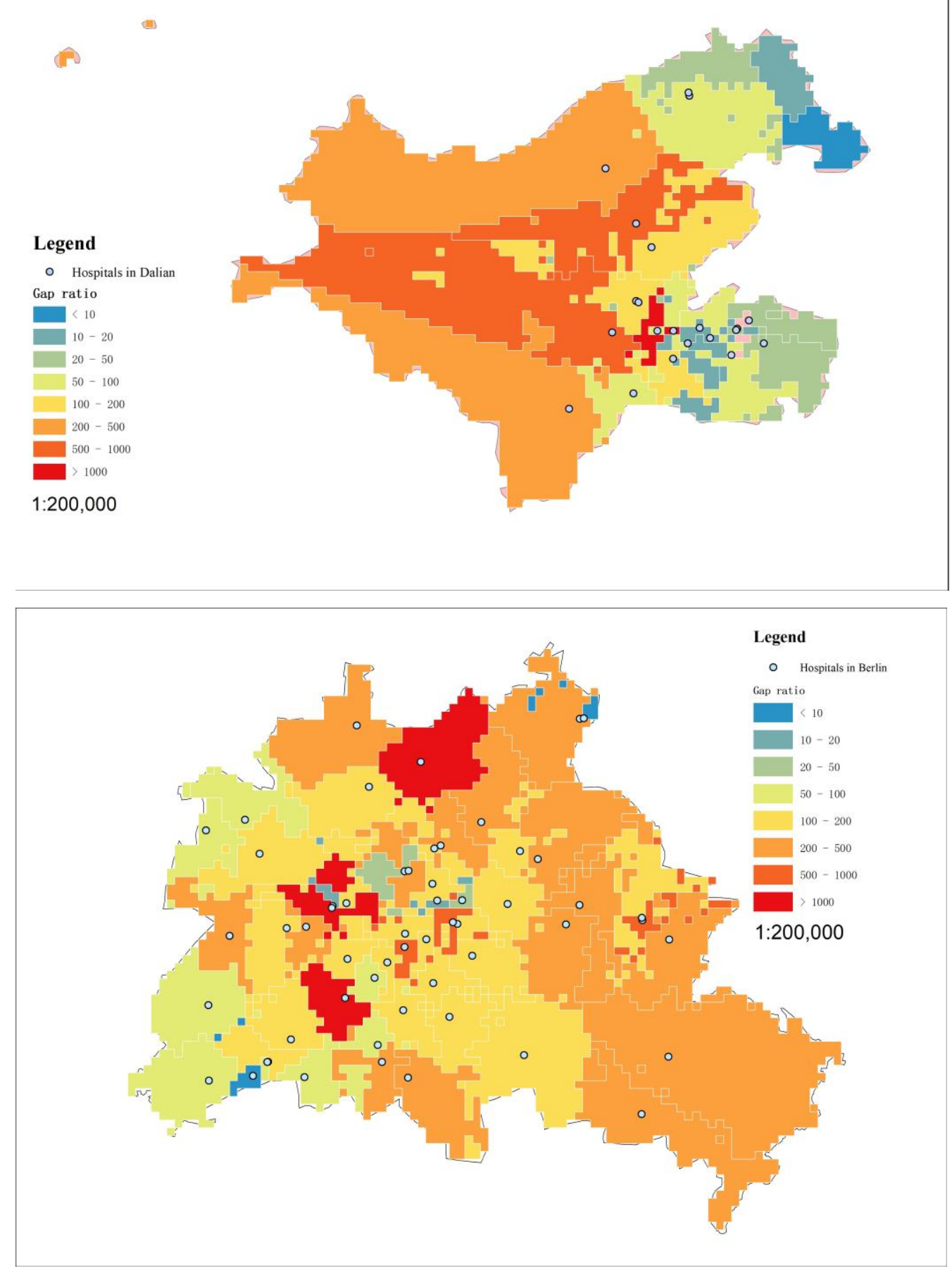

Figure 10 "Gap ratio" in Dalian and Berlin 
Table 2 Summary of "Gap ratio" in Dalian and Berlin

\begin{tabular}{lll}
\hline & Dalian & Berlin \\
\hline Weighted average “Gap ratio” & 445.2 & 253.2 \\
Gini Coefficient of “Gap ratio” & $53.1 \%$ & $52.7 \%$ \\
\hline
\end{tabular}

\section{Conclusions}

This paper introduces a novel method to demarcate the hospital service area based on the accessibility defined by the realistic travel time, and thus estimate the realistic demands of hospitals via calculation of total population within each service area. On that basis, the gaps between planned service levels and in Dalian and Berlin are examined. Our method offers a new angle to configure medical facilities, and has the potential to be transferred to the configuration of other public facilities as well.

From the perspective of the total amount of medical services, it is found that total number of hospital beds in Dalian is no lower than Berlin, or even higher from a per capita perspective. Yet the bottleneck remains as the hospitals in Dalian is fewer than half of Berlin. Therefore, it is argued that the total number of general hospitals in Dalian should be increased for improving the accessibility of medical service facilities in a regional level.

From the perspective of spatial distribution, the medical resources in Dalian are distributed rather unevenly in general, and the gaps between planned service level and realistic demands have been more notable in Dalian than in Berlin. A large number of hospitals with abundant medical resources are concentrated in the eastern part of the main urban area, namely Zhongshan District, Xigang District and Shahekou District. However, Ganjingzi District, the administrative district with the largest population and administrative area of Dalian, lacks correspondent medical service resources. It is obvious that realistic demands are huge, yet the planned service levels are relatively inferior. Thus, hospitals should be arranged more in the northern and western parts of the main urban area, so as to narrow the gaps between planned service levels and realistic demands.

\section{References}

BONDARENKO, M., KERR, D., SORICHETTA, A. \& TATEM, A. 2020. Census/projection-disaggregated gridded population datasets, adjusted to match the corresponding UNPD 2020 estimates, for 183 countries in 2020 using Built-Settlement Growth Model (BSGM) outputs.: WorldPop, University of Southampton, UK.

CHAI, Y.-W. \& LI, C.-J. 2019. Urban life cycle planning: From research to practice. City Plan. Rev, 43, 9-16.

LAN, W., LIAO, S. \& ZHAO, X. 2016. Exploration of Approaches and Factors of Healthy City Planning. Urban Planning International.

MENG, L. I. 2017. The Planning Strategies of a 15-minute Community Life Circle Based on Behaviors of Residents. Urban Planning Forum.

RONG, C. 2018. Spatial Governance Measures Of 15-minute Life Circle With A General Perspective. Planners.

SCOTT, L. M. \& JANIKAS, M. V. 2010. Spatial statistics in ArcGIS. Handbook of applied spatial analysis. Springer.

SONG, Z., CHEN, W., FENG, Y. \& WANG, L. 2010. Formulation of Public Facility Location Theory Framework and Literature Review. progress in geography, 29, 1499-1508.

TAO, Y. \& SHEN, Y. Spatial difference of medical facility accessibility and influencing factors:A comparative study of registered and floating populations in Shanghai. Progress in Geography.

ZHANG, D., CHEN, W., XUEPING, L. I. \& KONG, N. 2006. Research on the Code of Urban Community Public Service Facilities Plan and Its Implementation Unit:A Case of Wuhan. Urban Planning Forum.

ZHENG, Z., XIA, H., AMBINAKUDIGE, S., QIN, Y., LI, Y., XIE, Z., ZHANG, L. \& GU, H. 2019. Spatial accessibility to hospitals based on web mapping API: An empirical study in Kaifeng, China. Sustainability, 11, 1160. 